\title{
Development of Flexible Antimicrobial Packaging Materials against Campylobacter jejuni by Incorporation of Gallic Acid into Zein-Based Films
}

\author{
Derya Alkan, Levent Y. Aydemir, Iskender Arcan, Hatice Yavuzdurmaz, Halil I. Atabay, ${ }^{\dagger}$ Cagatay Ceylan, and \\ Ahmet Yemenicioğlu*
}

Department of Food Engineering, Faculty of Engineering, Izmir Institute of Technology, 35430, Gülbahçe Köyü, Urla, İzmir, Turkey

ABSTRACT: In this study, antimicrobial films were developed against Campylobacter jejuni by incorporation of gallic acid (GA) into zein-based films. The zein and zein-wax composite films containing GA between $2.5 \mathrm{and} 10 \mathrm{mg} / \mathrm{cm}^{2}$ were effective on different C. jejuni strains in a concentration-dependent manner. Zein and zein-wax composite films showed different release profiles in distilled water but quite similar release profiles at solid agar medium. Depending on incorporated GA concentration, $60-80 \%$ of GA released from the films, while the remaining GA was bound or trapped by film matrix. The GA at 2.5 and $5 \mathrm{mg} / \mathrm{cm}^{2} \mathrm{caused}$ a considerable increase in elongation $(57-280 \%)$ of all zein films and eliminated their classical flexibility problems. The zein-wax composite films were less flexible than zein films, but the films showed similar tensile strengths and Young's modulus. Scanning electron microscopy indicated different morphologies of zein and zein-wax composite films. This study clearly showed the good potential of zein and GA to develop flexible antimicrobial films against C. jejuni.

KEYWORDS: Zein, gallic acid, edible film, composite, antimicrobial, Campylobacter jejuni

\section{INTRODUCTION}

Campylobacters are present as commensals in the intestinal tracts of many warm-blooded animals including poultry, sheep, cattle, and swine. ${ }^{1}$ Campylobacteriosis is regarded as the most common cause of acute human bacterial enteritis worldwide. ${ }^{2}$ Thermophilic Campylobacter species such as Campylobacter jejuni and Campylobacter coli represent the most common species within the genus, as the former being the most frequent species that is responsible for the majority of cases. ${ }^{3}$ The incidence of intestinal disease associated with Campylobacter is considered to be as high as $1 \%$ of the population per year in many European countries and in the United States. ${ }^{2}$ It is generally thought that campylobacteriosis is mainly a foodborne disease, although there are other means of transmission to humans such as contact with domestic pets or farm animals. ${ }^{2}$ Case control studies imply that consumption of undercooked poultry meat and handling of the raw poultry meat are major sources of human infections. ${ }^{4}$ The similarities in genotypes among the isolates from animal, food, and human samples also supported that the transmission of C. jejuni occurs mainly from chickens in the farm to the retail chicken meat, and this is followed by cross-contamination of retail chicken meat and transmission to human. ${ }^{5}$ Chicken meat can be cross-contaminated during slaughter and dressing since pathogenic bacteria are usually present on the surface of the carcasses. ${ }^{6}$ Although there are several ways of reducing the contamination rates such as disinfection of crates and effective control measures at the slaughter during processing, a final decontamination step including chlorine dioxide, organic acids, trisodium phosphate, irradiation, UV light, heat, and high-intensity pulsed light would be very valuable. ${ }^{5-7}$

The active packaging is a rapidly developing technology that can be employed for controlling infectious foodborne outbreaks.
Different antimicrobial chemicals such as organic or inorganic acids, metals, alcohols, ammonium compounds, or amines have been incorporated into plastic packaging materials. ${ }^{8,9}$ However, because of the health concerns of the consumers and environmental problems associated with chemicals and plastics, extensive studies have been conducted to use natural bioactive agents including antimicrobial enzymes, bacteriocins, essential oils, and phenolic compounds in biodegradable or edible packaging materials. ${ }^{9,10}$ The use of phenolic compounds and extracts in active packaging attracts a particular interest since these compounds show potent antimicrobial and antioxidant activity in food systems and their intake can make a contribution to human health. ${ }^{10-12}$ Recent studies clearly showed the effectiveness of different phenolic extracts and pure phenolic compounds on C. jejuni. ${ }^{13-15}$ Gañan et al. ${ }^{13}$ tested 12 different phenolic compounds in phosphate-buffered saline medium at concentrations between 1 and $1000 \mathrm{mg} / \mathrm{mL}$ and identified gallic acid (GA) and $p$-hydroxybenzoic acid as potent inhibitors of $C$. jejuni. Klancnik et al. ${ }^{14}$ also investigated different plant extracts and pure phenolic compounds such as carnosic and rosmarinic acids on different bacteria and identified $C$. jejuni as more sensitive to phenolic inhibition than major Gram-negative bacteria including Escherichia coli O157:H7 and Salmonella infantis. Moreover, Nohynek et al. ${ }^{15}$ inhibited $C$. jejuni by using different berry phenolic extracts rich in ellagitannins. All of these reports in the literature suggested the good potential of using phenolic compounds to develop antimicrobial packaging materials against C. jejuni. However, studies in the literature about developing

Received: February 7, 2011

Accepted: September 10, 2011

Revised: September 9, 2011

Published: September 11, 2011 
edible antimicrobial films specifically against $C$. jejuni by use of phenolic compounds are scarce. In fact, it was only Arcan and Yemenicioglu ${ }^{16}$ who conducted preliminary antimicrobial tests with zein films containing different phenolic compounds and identified GA as an effective antimicrobial against pathogenic bacteria including $C$. jejuni. GA is also an effective plastisizer for zein films and eliminates their classical brittleness and flexibility problems, which caused great limitations in their widespread use for packaging applications. ${ }^{16}$ In this study, GA containing flexible zein films were developed specifically against $C$. jejuni by conducting detailed antimicrobial tests on its different strains, a chicken carcass isolate, and a standard human strain. Flexible composites of zein with carnauba wax were also prepared to obtain alternative film characteristics including release profiles, mechanical properties, and morphologies, which might be needed for different food applications. This study makes a contribution to develop novel active edible packaging systems, which could improve food quality and safety and reduce environmental concerns originating from plastics.

\section{MATERIALS AND METHODS}

Materials. Zein, GA, carnauba wax used in film making, and agar used in release tests were obtained from Sigma Chem Co. (St. Louis, MO). Glycerol was purchased from Merck (Darmstadt, Germany). Nutrient broth (No:2) and buffered peptone water were obtained from Oxoid Ltd. (Hampshire, United Kingdom). Nutrient agar used in antimicrobial tests was obtained by adding 1.4\% agar (Applichem, Darmstadt, Germany) in nutrient broth prepared according to the user's manual. All other chemicals were reagent grade. The fresh chicken samples, legs, necks, and breasts, from different commercial farms, were taken from a local butcher in Izmir and used in isolation studies. The samples were packed separately at aseptic condition, transferred to laboratory in ice boxes, and treated for isolation at the same day.

$C$. jejuni Strains. Two strains of $C$. jejuni were used in the present study. One of the strains was a reference strain isolated from human (C. jejuni NCTC12145). The other strain used was isolated for this study from chicken carcasses and phenotypically characterized as described earlier by Atabay and Corry. ${ }^{17}$ Identification of Campylobacter isolate at the species level was accomplished by $16 \mathrm{~S}$ rDNA sequencing. Briefly, genomic DNA was extracted and purified using Purelink Genomic DNA Mini Kit (Invitrogen, CA). The amplification was carried out as described by Mora et al., ${ }^{18}$ using universal forward and reverse primers: EGEI ( $5^{\prime}$-AGAGTTTGATCCTGGCTCAG-3') and EGEII ( $5^{\prime}$-CTAC GGCTACCTTGTTACCA-3') (Promega, Madison, WI). Polymerase chain reaction $(\mathrm{PCR})$ products were analyzed using $0.8 \%$ agarose gel electrophoresis (Minicell-Thermo EC320, Thermo Scientific, MA) at $90 \mathrm{~V}$ for $30 \mathrm{~min}$. The sequences obtained by a genetic analyzer (Applied Biosystems, model ABI 3130XL, CA) after amplification were compared and analyzed by using the basic local alignment search tool (BLAST, http://blast.ncbi.nlm.nih.gov/). According to the BLAST results, the isolate was identified as $C$. jejuni.

Film Making. Zein films were produced as described in Padgett et al. ${ }^{19}$ Briefly, $1.4 \mathrm{~g}$ of corn zein was dissolved with $8.1 \mathrm{~mL}$ of ethanol (97\%) by mixing at $200 \mathrm{rpm}$ with a magnetic stirrer for $25 \mathrm{~min}$. A $0.4 \mathrm{~mL}$ amount of glycerol was then added to the medium, and the temperature of the mixture was increased until it started to boil. The mixing was then ceased, and the film solution was boiled for $5 \mathrm{~min}$. After the mixture was cooled to room temperature, 21, 42, 84, and $168 \mathrm{mg}$ GA was added per $\mathrm{g}$ of film forming solutions to obtain $1.25,2.5,5$, and $10 \mathrm{mg} \mathrm{GA} \mathrm{per} \mathrm{cm}^{2}$ of dried films, respectively. The mixtures were then homogenized (Heidolph, Germany, rotor $\Phi=6.6 \mathrm{~mm}$ tip) at $10000 \mathrm{rpm}$ for $4 \mathrm{~min}$, and their $4.3 \mathrm{~g}$ portions were poured into glass templates $(\mathrm{W} \times \mathrm{L} \times \mathrm{H}$ :
$8.5 \mathrm{~cm} \times 8.5 \mathrm{~cm} \times 0.4 \mathrm{~cm})$. Zein-wax composite films were produced with this same procedure by adding carnauba wax $[5 \%(\mathrm{w} / \mathrm{w})$ of zein] into films after addition of glycerol (before initiation of heating). The films used in release tests and antimicrobial test were dried at $25^{\circ} \mathrm{C}$ for $19 \mathrm{~h}$ in an incubator, while films used in mechanical testing were dried at the same temperature for $48 \mathrm{~h}$ under $50 \%$ relative humidity using a controlled test cabinet (TK 120, Nüve, Turkey). The dried films were peeled from the glass templates carefully and used in different tests.

Antimicrobial Activity of Films against $C$. jejuni. Fifteen discs $(1.3 \mathrm{~cm}$ in diameter) were prepared from films by a cork borer under aseptic conditions. During tests, three discs were placed carefully onto each Petri dish containing nutrient agar that was inoculated with different $C$. jejuni strains. The inocula of microorganisms were prepared in peptone water using a $48 \mathrm{~h}$ culture of $C$. jejuni incubated at microaerophilic conditions at $37^{\circ} \mathrm{C}$ (achieved by jars of Anoxomat, Mart Microbiology, Holland). Before tests, the cell concentration was set to $1 \mathrm{McF}$ arland unit (corresponded to $3 \times 10^{8} \mathrm{cfu} / \mathrm{mL}$ ), and Petri dishes were inoculated by spread plate method by using $0.2 \mathrm{~mL}$ of culture diluted 1:10 with peptone water. The inoculated Petri dishes containing film discs were incubated at microaerophilic conditions at $37^{\circ} \mathrm{C}$ for $48 \mathrm{~h}$, and the diameter of the clear zones formed was measured by using a caliper. All antimicrobial tests were repeated three times, and results were expressed as average zone areas $\left(\mathrm{mm}^{2}\right)$.

Release Tests in Distilled Water. The release tests in distilled water were conducted to understand diffusion profiles of GA from different films and determine free (untrapped or unbound) GA content within the films. The tests were conducted by placing films $(4 \mathrm{~cm} \times$ $4 \mathrm{~cm})$ in distilled water at $4{ }^{\circ} \mathrm{C}$ in shaken $(80 \mathrm{rpm})$ Petri dishes. The tests last for 13 days, until reaching equilibrium or until an insignificant increase occurred in GA concentration by time. For sampling, $300 \mu \mathrm{L}$ of sample was taken at different time periods and assayed for total phenolic content by a spectrophotometer (Shimadzu, model 2450, Tokyo, Japan) using standard Folin-Ciocalteu method at $765 \mathrm{~nm} .{ }^{20}$ The calibration curve was prepared by using GA. The average of three measurements was used in calculations, and results were expressed as $\mathrm{mg}$ of GA released per $\mathrm{cm}^{2}$ of films $\left(\mathrm{mg} / \mathrm{cm}^{2}\right)$.

Release Tests in Solid Agar Medium. The test medium was prepared by pouring $15 \mathrm{~g}$ portions of $3 \%(\mathrm{w} / \mathrm{w})$ agar solution into mini disposable Petri dishes $(3.5 \mathrm{~cm}$ in diameter) and incubating dishes at room temperature for gel formation. The Petri dishes were then cooled down to $+4{ }^{\circ} \mathrm{C}$ before release tests. During release tests, the films $(1.5 \mathrm{~cm} \times$ $1.5 \mathrm{~cm}$ ) were placed between gel and Petri dish (below gel) by carefully removing one side of gel and putting film into midpoint of Petri dish. The Petri dishes were then incubated at $+4{ }^{\circ} \mathrm{C}$ for different time periods (up to 20 days) until reaching the equilibrium or until an insignificant increase occurred in GA concentration by time. At the end of each incubation period, after the films were removed, the contents of related Petri dishes were homogenized in a blender (Waring, United States) containing $40 \mathrm{~mL}$ of cold ethanol. The homogenates were then clarified by centrifugation at $15000 \mathrm{~g}$ for $15 \mathrm{~min}$, and clear supernatants were analyzed for GA content as described in release tests in destilled water. For each incubation period, the tests were conducted for three films placed in separate Petri dishes. Each sample was assayed for GA content for three times.

Fourier Transform Infrared (FTIR) Analysis of Films. The FTIR analysis was applied to determine potential hydrogen bond formation between zein and GA. For this purpose, the zein films were placed on the horizontal attenuated total reflectance sampling accessory (ZnSe crystal plate) of a FTIR spectrometer equipped with DTGS detector (Spectrum 100 Instrument, Perkin-Elmer Inc., Wellesley, MA). FTIR spectra of the samples were recorded between 4000 and $600 \mathrm{~cm}^{-1}$. Interferograms were averaged for 32 scans at $4 \mathrm{~cm}^{-1}$ resolution. The background spectrum was automatically subtracted from the spectra of the samples. Spectrum 100 software (Perkin-Elmer) was used for all data 


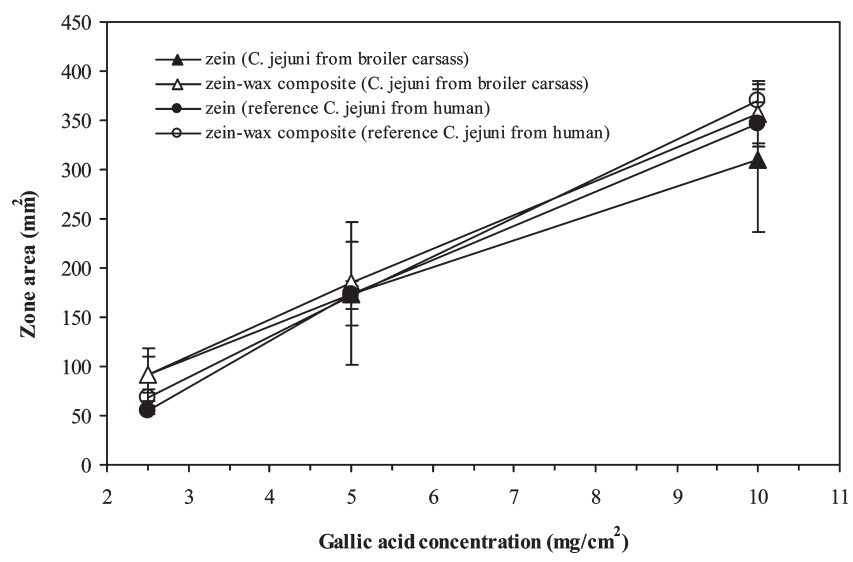

Figure 1. Antimicrobial activity of GA containing zein and zein-wax composite films on different $C$. jejuni strains.

manipulations. For each films, nine different scans $(n=9)$, which gave identical spectra, were performed. These replicates were averaged, and the averaged spectrum for each sample was then used for further data manipulation. The spectra were interactively baselined from two arbitrarily selected points. Finally, the spectra were normalized in specific regions for visual comparison of the control film and films containing GA. The spectra presented in the figures were normalized with respect to specific selected bands and are used only for illustrative purposes. However, in the measurement of the spectral parameters, each original baseline-corrected spectrum belonging to the corresponding control film and films containing GA were considered separately.

Mechanical Properties of Films. Tensile strength at break, elongation at break, and Young's modulus were determined using a Texture Analyzer TA-XT2 (Stable Microsystems, Godalming, United Kingdom) according to ASTM Standard Method D 882-02. ${ }^{21}$ Films were cut into $8 \mathrm{~mm}$ wide and $80 \mathrm{~mm}$ length strips. The initial grip distance was $50 \mathrm{~mm}$, and the crosshead speed was $500 \mathrm{~mm} / \mathrm{min}$. Five replicates of each film were tested.

Scanning Electron Microscopy (SEM). The photographs of film cross-sections and film thickness were determined by SEM (Philips XL 30S FEG, FEI Company, Netherlands). The films were prepared for SEM by crashing, following freezing in liquid nitrogen. The thickness of the films was measured from SEM cross-sectional views of films by using Scandium software (Olympus Soft Imaging Solutions).

Statistical Analysis. Results were analyzed for significance by the Fisher's protected least significant difference method. Differences were considered significant if $P<0.05$. In FTIR analysis, the differences between the control and the GA-containing film groups were compared using the Mann -Whitney U Test with the Matlab R2011a program. For FTIR results only, differences were considered significant if $P<0.01$.

\section{RESULTS AND DISCUSSION}

Antimicrobial Activity of Zein Films against $C$. jejuni. The effect of GA on $C$. jejuni isolated from broiler carcass was investigated by using $1.25-10 \mathrm{mg}$ GA per $\mathrm{cm}^{2}$ of films. The use of GA at $1.25 \mathrm{mg} / \mathrm{cm}^{2}$ did not give measurable zones on C. jejuni. However, GA between 2.5 and $10 \mathrm{mg} / \mathrm{cm}^{2}$ showed antimicrobial activity on the isolated bacteria in a concentrationdependent manner (Figure 1). At the studied concentration range, a 2-fold increase in GA concentration caused almost 1.8-3.0-fold increase in antimicrobial activity of films. The antimicrobial activity based on zone areas of zein and zein-wax composite films showed no statistically significant differences $(P>0.05)$. The zein and zein-wax composite films also showed

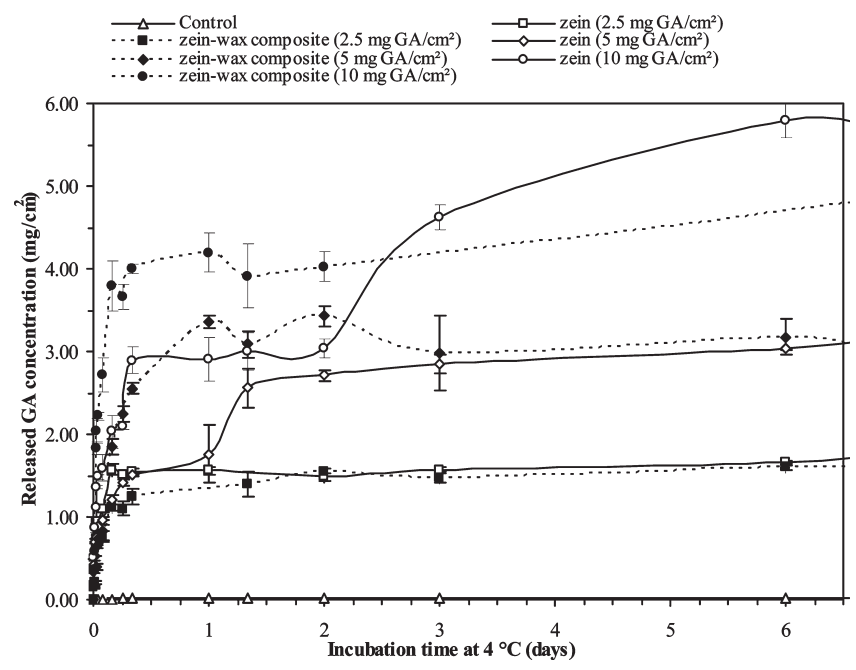

Figure 2. Release profiles of GA from zein and zein-wax composite films incubated in distilled water at $4{ }^{\circ} \mathrm{C}$.

quite similar antimicrobial activity on the reference $C$. jejuni strain from human. In the literature, studies related to development of antimicrobial edible films containing phenolic compounds specifically against $C$. jejuni were scarce. However, the results obtained in this work supported the previous findings of Gañan et al. ${ }^{13}$ who identified GA as a potent inhibitor of $C$. jejuni. Understanding of the exact mechanism of $C$. jejuni inhibition by GA needs further studies since the inactivation of bacteria by phenolic compounds may occur by multiple mechanisms including complex formation with cell walls, membrane disruption, inhibition of bacterial adhesion, or inactivation of bacterial enzyme systems. ${ }^{22}$ However, Nohynek et al. ${ }^{15}$ attributed the inhibitory action of GA on different Salmonella strains, Gramnegative bacteria as C. jejuni, to destruction of their outer membranes by this phenolic compound.

Release Tests. The release tests of zein and zein-wax composite films conducted in distilled water at $4{ }^{\circ} \mathrm{C}$ showed that the amount of released GA from films corresponded to $60-75 \%$ of the total GA incorporated into films (Figure 2). Thus, it is clear that the significant part of the GA incorporated into films released without being bind or trapped by the film matrix (Table 1). On the other hand, the remaining GA was retained within the film matrix since the phenolic compounds are capable to form H-bonding with proteins. ${ }^{23}$ Part of the GA might also be physically trapped within the hydrophobic zein clusters. The films containing $10 \mathrm{mg} / \mathrm{cm}^{2}$ GA showed the highest percentage of bind and/or trapped GA. It seemed that the GA molecules at this high concentration interacted with each other, and this caused some limited polymerization, which resulted with more GA binding and/or trapping.

On the other hand, the release tests in water with zein and zeinwax composite films showed that films containing $2.5 \mathrm{mg} / \mathrm{cm}^{2}$ GA showed similar release profiles, while GA in zein-wax composite films released more rapidly than the GA in zein films when phenolic concentration was increased to 5 or $10 \mathrm{mg} / \mathrm{cm}^{2}$. The zein-wax films containing $5 \mathrm{mg} / \mathrm{cm}^{2} \mathrm{GA}$ were thinner than zein films containing same amount of GA, but films containing $10 \mathrm{mg} / \mathrm{cm}^{2} \mathrm{GA}$ had quite similar thicknesses. Thus, the different release profiles of zein and zein-wax containing films at high GA concentration should not only be related in differences of film thickness. It appeared that the addition of wax caused some 
Table 1. Amount of GA Released from Films in Different Release Mediums

\begin{tabular}{|c|c|c|c|c|}
\hline \multicolumn{2}{|c|}{ film composition } & \multicolumn{2}{|c|}{$\begin{array}{l}\text { total released GA at equilibrium } \\
\qquad\left(\mathrm{mg} / \mathrm{cm}^{2}\right)\end{array}$} & \multirow[b]{2}{*}{$\begin{array}{c}\text { average film } \\
\text { thickness }(\mu \mathrm{m})\end{array}$} \\
\hline $\begin{array}{c}\mathrm{GA} \\
\left(\mathrm{mg} / \mathrm{cm}^{2}\right)\end{array}$ & $\begin{array}{l}\text { wax } \\
(\%)^{a}\end{array}$ & $\begin{array}{l}\text { release tests } \\
\text { in water }\end{array}$ & $\begin{array}{l}\text { release tests at } \\
\text { solid agar }\end{array}$ & \\
\hline & & & & $136.8 \pm 2.4 \mathrm{~b}$ \\
\hline 2.5 & & $1.86 \pm 0.11 \mathrm{a}(74)^{c}$ & $1.98 \pm 0.08 \mathrm{a}(79)$ & $123.2 \pm 1.3 c$ \\
\hline 2.5 & 5 & $1.68 \pm 0.13 \mathrm{a}(67)$ & $1.77 \pm 0.29 \mathrm{a}(71)$ & $133.1 \pm 1.7 \mathrm{~b}$ \\
\hline 5.0 & & $3.24 \pm 0.05 \mathrm{~b}(65)$ & $3.56 \pm 0.41 b(71)$ & $133.9 \pm 2.0 \mathrm{~b}$ \\
\hline 5.0 & 5 & $3.54 \pm 0.10 b(71)$ & $3.64 \pm 0.55 b(73)$ & $97.7 \pm 3.5 \mathrm{e}$ \\
\hline 10.0 & & $5.88 \pm 0.04$ c (59) & $6.47 \pm 0.46 c(65)$ & $171.4 \pm 20.0 \mathrm{a}$ \\
\hline 10.0 & 5 & $6.21 \pm 0.03 c(62)$ & $6.16 \pm 0.31 c(62)$ & $167.8 \pm 7.2 \mathrm{a}$ \\
\hline
\end{tabular}

${ }^{a}$ Wax content of films as percent of zein. ${ }^{b}$ Different letters in each column show significant difference at $P<0.05 .{ }^{c}$ Percentage of GA released from the films (the remaining GA was bound or trapped within the films).

morphological changes in films or differences in their swelling characteristics. Thus, differences in release profiles of zein and zein-wax composite films might also appear in liquid phase of film-coated solid and semisolid foods. To better understand the release characteristics of developed films, the release tests were also conducted at solid agar medium, which simulates semisolid or solid food systems (Figure 3). However, the release profiles of zein and zein-wax composite films at agar system were found quite similar with each other. It seemed that the diffusion of GA within the agar was very slow, and this minimized differences in diffusion rates of GA from films. In water, the release of GA was controlled mainly by diffusion coefficient of GA within the films and swelling characteristics of films. However, the concentration gradient of GA between film and agar and slow swelling rates of films at agar also become important factors effecting release rates of GA from films to agar. Further studies with original target food are needed to understand diffusion characteristics of GA from film to surface of solid or semisolid foods and within these foods. On the other hand, the release rates (GA released per day) of GA from films tested in agar system increased considerably as GA content of films was increased. In fact, the total amount of GA released by $2.5 \mathrm{mg} / \mathrm{cm}^{2}$ GA containing zein and zein-wax composite films at agar system in 10 days $\left(1.8-1.9 \mathrm{GA}\right.$ per $\left.\mathrm{cm}^{2}\right)$ was reached in almost $1.5-3$ and 0.5 days for 5 and $10 \mathrm{mg} / \mathrm{cm}^{2} \mathrm{GA}$ containing films, respectively. These results clearly showed the importance of choosing suitable concentrations of GA in films depending on the magnitude of risks associated with the food and length of targeted shelf life.

Mechanical Properties of the Films. The results of mechanical tests for zein and zein-wax composite films incorporated with GA are given in Table 2. As expected, the control zein films were highly brittle and showed very little elongations. The incorporation of wax into films alone (without GA) reduced and increased the tensile strength and Young's modulus of films, respectively, but it did not cause any statistically significant change in film elongation $(P>0.05)$. The reduced tensile strength but unchanged film elongation by wax addition into control films lacking GA suggested that the incorporation of wax and formation of the composite structure might disrupt and weakened the continuous zein film matrix. In the literature, data about mechanical properties of zein-carnauba wax composites are scarce, but

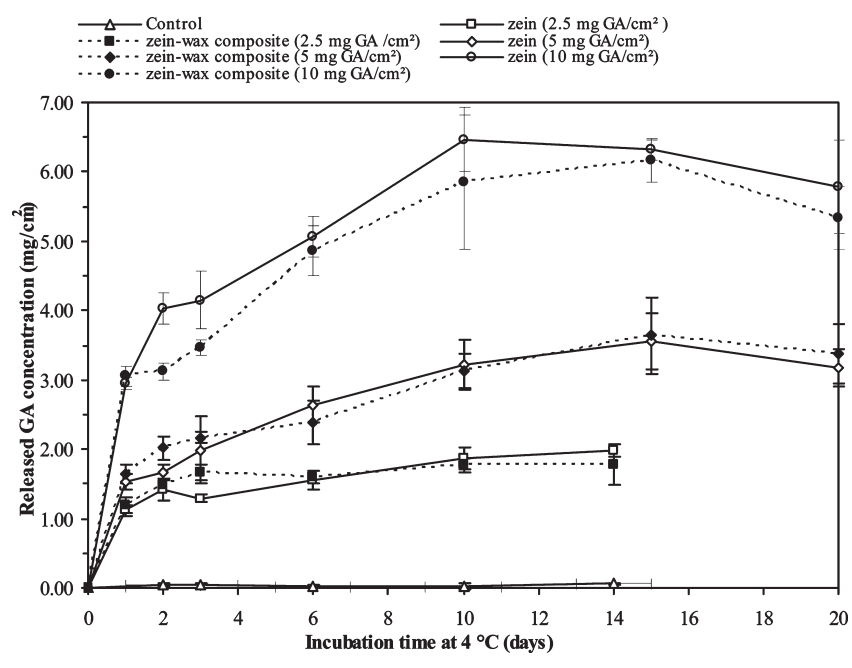

Figure 3. Release profiles of GA from zein and zein-wax composite films incubated at solid agar medium at $4{ }^{\circ} \mathrm{C}$.

Table 2. Mechanical Properties of Different Zein Films

\begin{tabular}{ccccc}
\multicolumn{2}{c}{ film composition } & & & \\
\cline { 1 - 1 } $\begin{array}{c}\text { GA (mg/ } \\
\left.\mathrm{cm}^{2}\right)\end{array}$ & $\begin{array}{c}\text { wax } \\
(\%)^{a}\end{array}$ & $\begin{array}{c}\text { tensile strength } \\
(\mathrm{MPa})\end{array}$ & $\begin{array}{c}\text { Young's modulus } \\
(\mathrm{MPa})\end{array}$ & $\begin{array}{c}\text { elongation } \\
(\%)\end{array}$ \\
& & $7.25 \pm 2.00 \mathrm{a}^{\mathrm{b}}$ & $3.81 \pm 0.61 \mathrm{~b}$ & $4 \pm 1 \mathrm{e}$ \\
& 5 & $4.05 \pm 0.95 \mathrm{~b}$ & $4.33 \pm 0.62 \mathrm{a}$ & $2 \pm 0 \mathrm{e}$ \\
2.5 & & $0.18 \pm 0.12 \mathrm{c}$ & $0.03 \pm 0.01 \mathrm{c}$ & $141 \pm 15 \mathrm{c}$ \\
2.5 & 5 & $0.16 \pm 0.01 \mathrm{c}$ & $0.04 \pm 0.0 \mathrm{c}$ & $57 \pm 9 \mathrm{~d}$ \\
5.0 & & $0.02 \pm 0.0 \mathrm{c}$ & $0.01 \pm 0.01 \mathrm{c}$ & $280 \pm 21 \mathrm{a}$ \\
5.0 & 5 & $0.04 \pm 0.0 \mathrm{c}$ & $0.01 \pm 0.0 \mathrm{c}$ & $221 \pm 15 \mathrm{~b}$ \\
10.0 & & $0.06 \pm 0.01 \mathrm{c}$ & $0.04 \pm 0.0 \mathrm{c}$ & $13 \pm 3 \mathrm{e}$ \\
10.0 & 5 & $0.08 \pm 0.0 \mathrm{c}$ & $0.06 \pm 0.0 \mathrm{c}$ & $3 \pm 0 \mathrm{e}$
\end{tabular}

${ }^{a}$ Wax content of films as percent of zein. ${ }^{b}$ Different letters in each column show significant difference at $P<0.05$.

Sohail et al., ${ }^{24}$ who incorporated paraffin wax into casein-zein hydrolizate films, reported a reduced tensile strength but increased elongation and flexibility of these films. In contrast, Chick and Hernandez ${ }^{25}$ reported an increased tensile strength but reduced film elongation after incorporating carnauba or candelilla wax into casein films. These reports showed the complexity of mechanical changes in protein-wax composite structures. It appears that the mechanical changes in composite systems could be highly variable depending on amounts and molecular properties of each constituent in the mixture and degree of their compatibility and interactions within the films. On the other hand, the addition of GA at 2.5 or $5 \mathrm{mg} / \mathrm{cm}^{2}$ into films eliminated the classical brittleness problem of zein films and caused statistically significant increases in film elongation. In general, the zein films containing GA showed higher elongation than zein-wax composite films containing similar amounts of GA. It seemed that the GA did not interact with the wax particles and aggregates distributed within the composite films, and this caused a limited reduction in its plastisizing effect. However, it is worth reporting that the zein films containing GA were very sticky and hard to peel from the plates after casting. The stickiness is also a disadvantage during handling of films for food applications. The addition of wax reduced the stickiness 

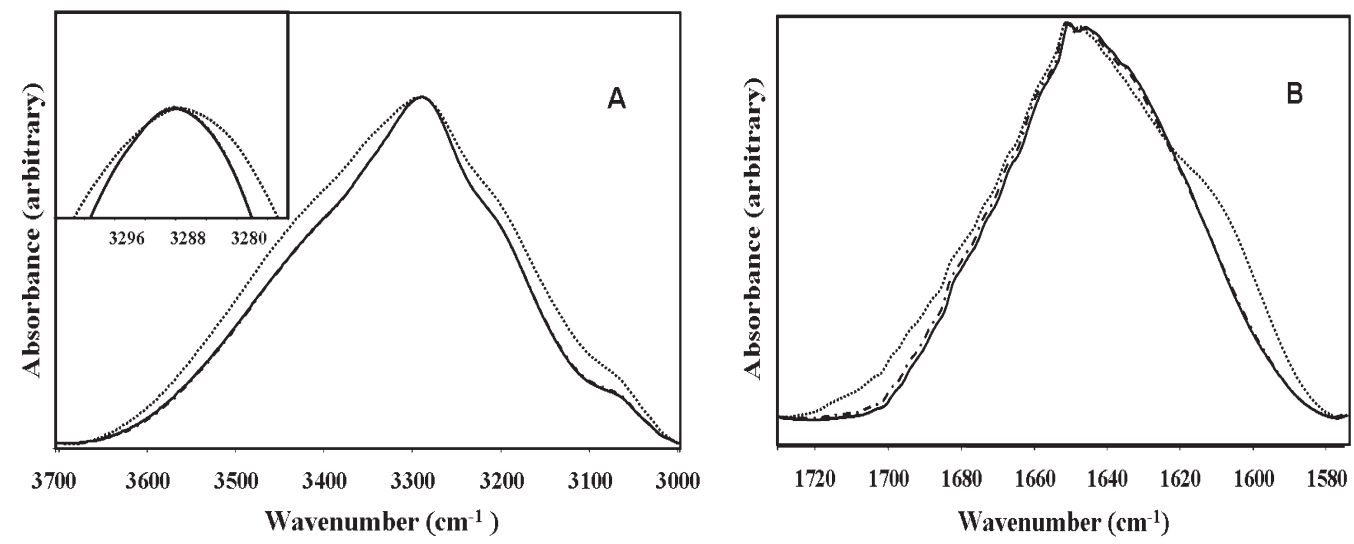

Figure 4. Average spectra of control film (-) and zein film containing $0.25(-\cdot-)$ or $2.5 \mathrm{mg} / \mathrm{cm}^{2} \mathrm{GA}(\cdot \ldots)$ (A) in the amide A spectral region (the spectra were baselined and then normalized with respect to the band at $3287 \mathrm{~cm}^{-1}$ ) and (B) in the amide I spectral region (the spectra were baselined and then normalized with respect to the band at $1651 \mathrm{~cm}^{-1}$ ).

considerably and let films peeled from plates easily. On the other hand, no statistically significant differences were determined between tensile strength and Young's modulus of zein and zein-wax composites containing GA $(P>0.05)$. The use of GA at $5 \mathrm{mg} / \mathrm{cm}^{2}$ gave the most elastic films, which showed elongation over $200 \%$. In contrast, a further increase of GA concentration to $10 \mathrm{mg} / \mathrm{cm}^{2}$ reduced elongations of films dramatically. It seemed that too much GA within the films caused polymerization of this phenolic compound and loss of its plasticizing effect. These results confirmed our recent findings about plasticizing effect of GA and different phenolic compounds in zein films. ${ }^{16}$ Further studies are needed to understand the exact mechanism of increased film flexibility by addition of GA. However, it is known that the brittleness and lack of flexibility in zein films are due to the hydrophobic interactions that keep the zein molecules together to maintain film integrity. ${ }^{26}$ Most plasticizers owe their positive effects on film flexibility to their hydroxyl groups, which form hydrogen bonds with polymers and increase the free volume of film matrix. ${ }^{27}$ Thus, it seems that the plasticizing effect of GA is related to its ability to form H-bonding with its hydroxyl and -carboxyl groups to peptide amino or carbonyl groups of zein to form a weak flexible network within the films and increase of film hydrophilicity.

In the literature, many studies have been conducted to plasticize highly brittle and nonflexible zein films and improve their mechanical properties by addition of different ingredients such as organic acids, sugars, alcohols, fatty acids, and different synthetic polymers, cross-linkers, or plasticizers. ${ }^{28-33}$ However, none of these studies provided an effective solution to flexibility and brittleness problems by use of natural bioactive compounds. Recently, the plasticizing effects of different phenolic compounds in zein films have been investigated by Arcan and Yemenicioglu. ${ }^{16}$ In that study, because of their sufficient number of hydroxyl groups and good solubility within zein films phenolic compounds like GA, (+)-catechin, $p$-hydroxy benzoic acid, and ferulic acid showed effective plasticizing effect within zein films, while flavone and quercetin were found ineffective plasticizers due to their lack of hydrogen-bonding hydroxyl groups and insolubility within the films, respectively. ${ }^{16}$ In the literature, positive or negative effects of phenolic compounds have been reported in mechanical properties of different edible films. For example, $\mathrm{Ou}$ et al. $^{34}$ reported a limited increase in elongation of soy protein films by addition of ferulic acid, but this phenolic acid effectively increased the tensile strengths of films.
$\mathrm{Ku}$ et al. ${ }^{35}$ incorporated the phenolic compound catechin into a carbohydrate film from agar extracted from Gelidium corneum but did not determine any plasticizing effect of catechin. In contrast, Emmambux et al. ${ }^{36}$ incorporated condensed tannins like tannic acid (TA) into films from sorghum kafirin, a zein like prolamin, reported an antiplasticizing effect of this phenolic compound after determining a reduced elongation, but increased tensile strength and Young's modulus of the films. The antiplasticizing effect of TA could be due to its molecular properties, since this phenolic compound contains too many hydroxyl groups, and it binds proteins very tightly to reduce their mobility within the film matrix.

FTIR Analysis of the Films. FTIR spectroscopy was employed to analyze the possible hydrogen bonds in control films and films containing 0.25 or $2.5 \mathrm{mg} / \mathrm{cm}^{2}$ GA. The zein film containing GA at $0.25 \mathrm{mg} / \mathrm{cm}^{2}$ was tested to see the spectral changes in films containing GA but lacking the desired flexibility. The films containing 5 and $10 \mathrm{mg} / \mathrm{cm}^{2}$ GA could not be analyzed, since the peaks obtained for these films originating from GA dominated the whole spectra, making it impossible to investigate the effects of possible hydrogen bond formation on the protein structure. The average FTIR spectra of control films and films containing different concentrations of GA were given in Figure 4. In Figure 4A,B showing amide A spectral region originating mainly form $\mathrm{N}-\mathrm{H}$ stretching and amide I spectral regions originating mainly from $\mathrm{C}=\mathrm{O}$ stretching, respectively, the increased bandwidth of $2.5 \mathrm{mg} / \mathrm{cm}^{2}$ GA containing films indicated hydrogen bond formation within these films. ${ }^{37-39}$ In contrast, the lack of any increase in bandwidth of $0.25 \mathrm{mg} / \mathrm{cm}^{2}$ GA containing films, which showed no increase in flexibility, suggested an insufficient number of hydrogen bond formation within these films. A band shift in the amide A band toward lower wave numbers also indicates the hydrogen bond formation. ${ }^{38,40}$ According to our results, the band at $3288.14 \pm 0.12 \mathrm{~cm}^{-1}$ for control film shifted down to $3287.98 \mathrm{~cm}^{-1} \pm 0.10$ with the addition of $0.25 \mathrm{mg} / \mathrm{cm}^{2} \mathrm{GA}(P=0.0078)$ and down to 3287.51 $\pm 0.30 \mathrm{~cm}^{-1}(P<0.001)$ with the addition of $2.5 \mathrm{mg} / \mathrm{cm}^{2} \mathrm{GA}$ into films. Thus, these data supported our hypothesis of a relation between increased film flexibility and formation of $\mathrm{H}$ bonding within zein films by the addition of GA. However, further studies are needed to investigate the effect of GA on molecular properties of zein and fully characterize the factors causing increased flexibility of films. 

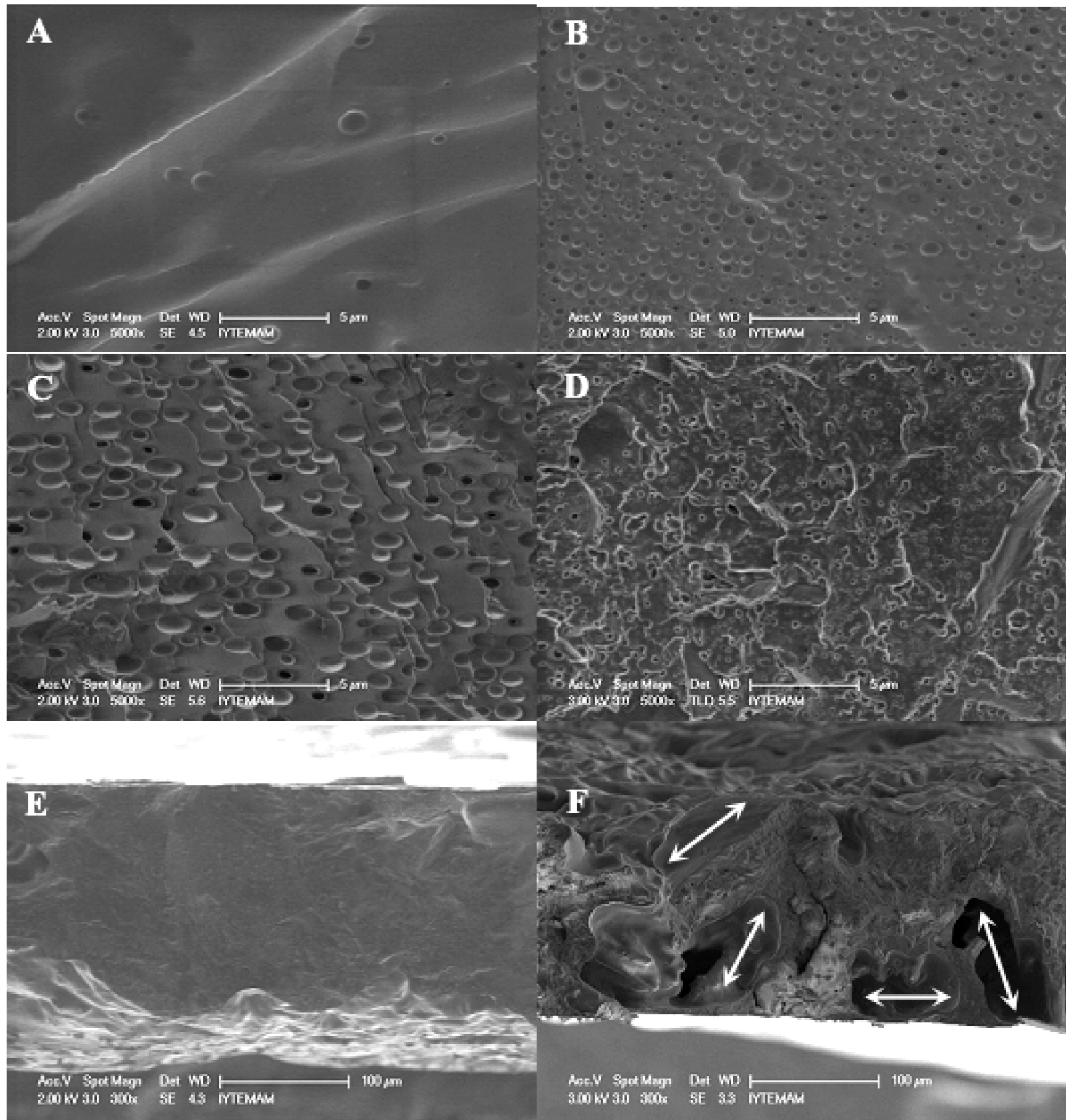

Figure 5. SEM photographs of different film cross-sections. (A) Control zein film, (B) $5 \mathrm{mg} / \mathrm{cm}^{2}$ GA containing zein film, (C) control zein-wax composite film, (D) $5 \mathrm{mg} / \mathrm{cm}^{2}$ GA containing zein-wax composite film, (E) $10 \mathrm{mg} / \mathrm{cm}^{2}$ GA containing zein film, and (F) $10 \mathrm{mg} / \mathrm{cm}{ }^{2} \mathrm{GA}$ containing zein-wax composite film. Magnifications were $5000 \times$ for A-D and $300 \times$ for E and F. Arrows indicate cracks and cavities.

SEM Images of Films. The SEM images of control zein and zein-wax composite films and GA containing zein and zein-wax composite films are given in Figure 5A-F. The cross-section images showed the morphological changes in films by addition of GA or wax (Figure $5 \mathrm{~A}-\mathrm{C}, 5000 \times$ ). It is clear that the addition of GA or wax alone into films increased the film porosity. Moreover, it is also observed that the addition of wax alone caused formation of larger pores than addition of GA alone into zein films. On the other hand, in films containing both GA and wax, the morphology was more complex since films contained high number of pores, and cracks and cavities with varying sizes, and heterogeneously distributed wax aggregates (Figure 5D, $5000 \times$ ). In particular, $10 \mathrm{mg} / \mathrm{cm}^{2}$ GA containing zein-wax films the sizes of pores were considerably greater than those of zein films containing same amount of GA (Figure 5E,F, 300×). In fact, some large crack and cavities were observed within these GA containing zein-wax composite films (see arrows in Figure 5F). These results showed the different morphologies of zein and zein-wax composite films and explained the higher relase rates of GA in distilled water from zein-wax composite films than the zein films (containing 5 and $10 \mathrm{mg} / \mathrm{cm}^{2} \mathrm{GA}$ ).

In conclusion, this work clearly showed the good potential of GA as an antimicrobial compound against different strains of C. jejuni. The plastisizing effect of GA in zein films enabled production of flexible edible films having antimicrobial activities. The incorporation of wax with GA formed less sticky, thus, more easily peeled and handled films having similar antimicrobial effectiveness and release profiles in agar but showing differences in release profiles in water, mechanical properties, and morphologies. Further studies are continuing to develop novel active packaging applications against $C$. jejuni by using zein and zeinwax composite films and coatings and test of their antimicrobial performance in food systems. 


\section{AUTHOR INFORMATION}

\section{Corresponding Author}

*Tel: 90(232)7506292. Fax: 90(232)7506196. E-mail: ahmetyemenicioglu@iyte.edu.tr.

\section{Present Addresses}

${ }^{\dagger}$ Department of Medical Microbiology, Faculty of Medicine, Sifa University, Ankara Caddesi, No. 45, Bornova, 35100, Izmir, Turkey.

\section{Funding Sources}

This work (no. 108M353) was funded by The Scientific and Technical Research Council of Turkey (TÜBITTAK).

\section{ACKNOWLEDGMENT}

We thank the Materials Research Center and Biotechnology and Bioengineering Research and Applications Center in Izmir Institute of Technology for enabling use of their facilities.

\section{REFERENCES}

(1) Skirrow, M. B. Diseases due to Campylobacter, Helicobacter and related bacteria. J. Comp. Pathol. 1994, 111, 113-149.

(2) Humphrey, T.; O'Brien, S.; Madsen, M. Campylobacters as zoonotic pathogens: A food production perspective. Int. J. Food Microbiol. 2007, 117, 237-257.

(3) Sails, A. D.; Fox, A. J.; Bolton, F. J.; Wareing, D. R. A.; Greenway, D. L. A.; Borrow, R. Development of a PCR ELISA assay for the identification of Campylobacter jejuni and Campylobacter coli. Mol. Cell. Probes 2001, 15, 291-300.

(4) Corry, J. E. L.; Atabay, H. I. Poultry as a source of Campylobacter and related organisms. J. Appl. Microbiol. 2001, 90, 96S-114S.

(5) Zorman, T.; Heyndrickx, M.; Uzunovic-Kamberovic, S.; Mozina, S. S. Genotyping of Campylobacter coli and C. jejuni from retail chicken meat and humans with campylobacteriosis in Slovenia and Bosnia and Herzegovina. Int. J. Food Microbiol. 2006, 110, 24-33.

(6) James, C.; James, S. J.; Hannay, N.; Purnell, G.; Barbedo-Pinto, C.; Yaman, H.; Araujo, M.; Gonzalez, M. L.; Calvo, J.; Howell, M.; Corry, J. E. L. Decontamination of poultry carcasses using steam or hot water in combination with rapid cooling, chilling or freezing of carcass surfaces. Int. J. Food Microbiol. 2007, 114, 195-203.

(7) Haughton, P. N.; Lyng, J. G.; Morgan, D. J.; Cronin, D. A.; Fanning, S.; Whyte, P. Efficacy of high-intensity pulsed light for the microbiological decontamination of chicken, associated packaging, and contact surfaces. Foodborne Pathog. Dis. 2011, 8, 109-117.

(8) Appendini, P.; Hotchkiss, J. H. Review of antimicrobial food packaging. Innovative Food Sci. Emerging Technol. 2002, 3, 113-126.

(9) Suppakul, P.; Miltz, J.; Sonneveld, K.; Bigger, S. W. Active packaging technologies with an emphasis on antimicrobial packaging and its applications. J. Food Sci. 2003, 68, 408-420.

(10) Coma, V. Bioactive packaging technologies for extended shelf life of meat-based products. Meat Sci. 2008, 78, 90-103.

(11) Crespy, V.; Williamson, G. A review of the health effects of green tea catechins in in vivo animal models. J. Nutr. 2004, 134, 3431S-3440S

(12) Komes, D.; Horzic, D.; Belscak, A.; Ganic, K. K.; Vulic, I. Green tea preparation and its influence on the content of bioactive compounds. Food Res. Int. 2010, 43, 167-176.

(13) Gañan, M.; Martínez-Rodríguez, A. J.; Carrascosa, A. V. Antimicrobial activity of phenolic compounds of wine against Campylobacter jejuni. Food Control 2009, 20, 739-742.

(14) Klancnik, A.; Piskernik, S.; Jergek, B.; Mozina, S. S. Evaluation of diffusion and dilution methods to determine the antibacterial activity of plant extracts. J. Microbiol. Methods 2010, 81, 121-126.
(15) Nohynek, L. J.; Alakomi, H. L.; Kahkonen, M. P.; Heinonen, M.; Helander, K. M.; Oksman-Caldentey, K. M.; Puupponen-Pimia, R. H. Berry phenolics: Antimicrobial properties and mechanisms of action against severe human pathogens. Nutr. Cancer 2006, 54, 18-32.

(16) Arcan, I.; Yemenicioğlu, A. Incorporating phenolic compounds opens a new perspective to use zein films as flexible bioactive packaging materials. Food Res. Int. 2011, 44, 550-556.

(17) Atabay, H. I.; Corry, J. E. L. The prevalence of campylobacters and arcobacters in broiler chickens. J. Appl. Microbiol. 1997, 83, 619-626.

(18) Mora, D.; Fortina, M. G.; Nicastro, G.; Parini, C.; Manachini, P. L. Genotypic characterization of thermophilic bacilli: A study on new soil isolates and several reference strains. Res. Microbiol. 1998, 149, 711-722.

(19) Padgett, T.; Han, I. Y.; Dawson, P. L. Incorporation of foodgrade antimicrobial compounds into biodegradable packaging films J. Food Prot. 1998, 61, 1330-1335.

(20) Singleton, V. L.; Rossi, J. A. Colorimetry of total phenolics with phosphomolybdic-phosphotungstic acid reagents. Am. J. Enol. Vitic. 1965, 16, 144-158.

(21) ASTM. Standard test method for tensile properties of thin plastic sheeting-D882-02. Annual Book of American Standard Testing Methods; ASTM: Philadelphia, PA, 2002.

(22) Cowan, M. M. Plant products as antimicrobial agents. Clin. Microbiol. Rev. 1999, 12, 564-582.

(23) Damodaran, S. Amino acids, peptides and proteins. In Food Chemistry, 3rd ed.; Fennema, O. R., Ed.; CRC Press Taylor \& Francis Group: New York, 1996; p 321.

(24) Sohail, S. S.; Wang, B.; Biwas, M. A. S.; Oh, J. Physical, morpological, and barrier properties of edible casein films with wax applications. J. Food Sci. 2006, 71, 255-259.

(25) Chick, J.; Hernandez, R. J. Physical, thermal, and barrier characterization of casein-wax-based edible films. J. Food Sci. 2002, 67, 1073-1079.

(26) Guo, Y. C.; Liu, Z. D.; An, H.J.; Li, M. Q.; Hu, J. Nano-structure and properties of maize zein studied by atomic force microscopy. J. Cereal Sci. 2005, 41, 277-281.

(27) Sothornvit, R.; Krochta, J. M. Plasticizers in edible films and coatings. In Innovations in Food Packaging; Jung, H. H., Ed.; Academic Press: London, 2005; pp 403-433.

(28) Lawton, J. W. Plasticizers for zein: Their effect on tensile properties and water absorption of zein films. Cereal Chem. 2004, $81,1-5$.

(29) Ghanbarzadeh, B.; Oromiehie, A. R.; Musavi, M.; D-Jomeh, Z. E.; Rad, E. R.; Milani, W. Effect of plasticizing sugars on rheological and thermal properties of zein resins and mechanical properties of zein films. Food Res. Int. 2006, 39, 882-890.

(30) Lai, H. M.; Padua, G. W. Properties and microstructure of plasticized zein films. Cereal Chem. 1997, 74, 771-775.

(31) Woods, K. K.; Selling, G. W.; Cooke, P. H. Compatible blends of zein and polyvinylpyrrolidone. J. Polym. Environ. 2009, 17, 115-122.

(32) Kim, S.; Sessa, D. J.; Lawton, J. W. Characterization of zein modified with a mild cross-linking agent. Ind. Crop. Prod. 2004, 20, 291-300.

(33) Sessa, D. J.; Mohamed, A.; Byars, J. A. Chemistry and physical properties of melt-processed and solution-cross-linked corn zein J. Agric. Food Chem. 2008, 56, 7067-7075.

(34) Ou, S.; Wang, Y.; Tang, S.; Huang, C.; Jackson, M. G. Role of ferulic acid in preparing edible films from soy protein isolate. J. Food Eng. 2005, 70, 205-210.

(35) Ku, K.-J.; Hong, Y.-H.; Song, K. B. Mechanical properties of a Gelidium corneum edible film containing catechin and its application in sausages. J. Food Sci. 2008, 73, C217-C221.

(36) Emmambux, M. N.; Stading, M.; Taylor, J. R. N. Sorghum kafirin film property modification with hydrolysable and condensed tannins. J. Cereal Sci. 2004, 40, 127-135.

(37) Cakmak, G.; Togan, I.; Severcan, F. 17[ $\beta]$-Estradiol induced compositional, structural and functional changes in rainbow trout liver, 
revealed by FT-IR spectroscopy: A comparative study with nonylphenol. Aquat. Toxicol. 2006, 77, 53-63.

(38) Mohammed-Ziegler, I.; Billes, F. Vibrational spectroscopic calculations on pyrogallol and gallic acid. J. Mol. Struct. THEOCHEM 2002, 618, 259-265.

(39) He, L.; Mu, C.; Shi, J.; Zhang, Q.; Shi, B.; Lin, W. Modification of collagen with a natural cross-linker, procyanidin. Int. J. Biol. Macromol. 2011, 48, 354-359.

(40) Maréchal, Y.; Chanzy, H. The hydrogen bond network in I [beta] cellulose as observed by infrared spectrometry. J. Mol. Struct. 2000, 523, 183-196. 